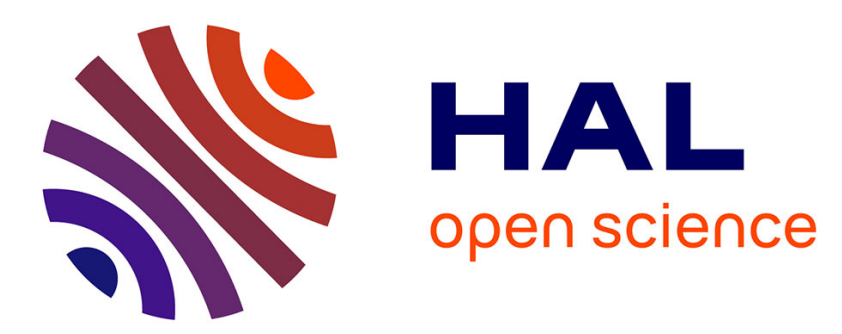

\title{
Double hybrids and time-dependent density functional theory: An implementation and benchmark on charge transfer excited states
}

Alistar Ottochian, Carmela Morgillo, Ilaria Ciofini, Michael Frisch, Giovanni Scalmani, Carlo Adamo

\section{To cite this version:}

Alistar Ottochian, Carmela Morgillo, Ilaria Ciofini, Michael Frisch, Giovanni Scalmani, et al.. Double hybrids and time-dependent density functional theory: An implementation and benchmark on charge transfer excited states. Journal of Computational Chemistry, 2020, 10.1002/jcc.26170 . hal-02879256

\section{HAL Id: hal-02879256 https://hal.science/hal-02879256}

Submitted on 10 Nov 2020

HAL is a multi-disciplinary open access archive for the deposit and dissemination of scientific research documents, whether they are published or not. The documents may come from teaching and research institutions in France or abroad, or from public or private research centers.
L'archive ouverte pluridisciplinaire HAL, est destinée au dépôt et à la diffusion de documents scientifiques de niveau recherche, publiés ou non, émanant des établissements d'enseignement et de recherche français ou étrangers, des laboratoires publics ou privés. 


\title{
Double Hybrids and Time-Dependent Density Functional Theory: an Implementation and Benchmark on Charge Transfer Excited States
}

\author{
Alistar Ottochian ${ }^{1}$, Carmela Morgillo ${ }^{1}$, Ilaria Ciofini ${ }^{1}$, Michael J. Frisch ${ }^{2}$, Giovanni \\ Scalmani $^{2^{*}}$, Carlo Adamo ${ }^{1,3^{*}}$ \\ Chimie ParisTech, PSL Research University, CNRS, Institute of Chemistry for Life and \\ Health Sciences, 11, rue Pierre et Marie Curie, F-75005 Paris, France; Gaussian, Inc. 340 \\ Quinnipiac St., Bldg. 40, Wallingford, Connecticut, 06492 USA; Institut Universitaire de \\ France, 103 Boulevard Saint Michel, F-75005 Paris, France,
}

\begin{abstract}
In this paper we present the implementation and benchmarking of a Time Dependent Density Functional Theory approach in conjunction with Double Hybrid (DH) functionals. We focused on the analysis of their performance for through space charge-transfer (CT) excitations which are well known to be very problematic for commonly used functionals, such as global hybrids.

Two different families of functionals were compared, each of them containing pure, hybrid and double-hybrid functionals. The results obtained show that, beside the robustness of the implementation, these functionals provide results with an accuracy comparable to that of adjusted range-separated functionals, with the relevant difference that for DHs no parameter is tuned on specific compounds thus making them more appealing for a general use. Furthermore, the algorithm described and implemented is characterized by the same computational cost scaling as that of the ground state algorithm employed for MP2 and double hybrid DFAs.
\end{abstract}

1)Chimie-ParisTech; 2) Gaussian; 3) IUF

*) corresponding author: carlo.adamo@chimie-paristech.fr; giovanni@gaussian.com 


\section{Introduction}

Approaches rooted in Density Functional Theory (DFT) nowadays provide valuable insights for the understanding of a wide variety of chemical problems as witnessed by their widespread use in the last two decades ${ }^{1-3}$. Their success mainly relies on a very favorable cost to accuracy ratio enabling the description of ground state properties of relatively large molecular systems.

Nonetheless the accuracy reached using DFT approaches strongly depends on the nature of the density functional approximation (DFA) used and, in particular, on the expression of the contribution to exchange and correlation energies ${ }^{1,2}$. For the time being, one of the most sophisticated and accurate DFAs for ground state properties are represented by the so called Double Hybrid (DH) functionals casting in the DFA expression both Hartree-Fock (HF) exchange and a second order perturbative contribution computed with Kohn-Sham orbitals to the correlation ${ }^{4-6}$. These functionals thus introduce an explicit dependence of the energy expression on both occupied and virtual orbitals. Several papers in literature, following the seminal works of $\operatorname{Truhlar}^{7}$ and Grimme ${ }^{6}$, have demonstrated their rigorous theoretical foundation $^{4-12}$.

If these functionals have proven to be extremely performant for many ground state properties (such as thermochemistry or the description of weak interacting systems ${ }^{13-14}$ ) nonetheless their largest limitation is the computational cost associated to the evaluation of their non-local correlation contribution. Indeed, even if scaling techniques may reduce the computational effort without altering their accuracy, DHs, with a formal scaling of $\mathcal{O}\left(N^{4}\right)$ at best, still remain expensive when compared to 'standard' global or range separated hybrids DFAs.

This situation is even more involved when considering the use of DHs for the treatment of excited state. Grimme and Neese proposed in $2007^{15}$ a Time Dependent formulation in the context of the Tamm-Dancoff approximation for DHs resting on a standard linear response formulation corrected by a second-order perturbative term derived from the Head-Gordon's CIS(D) approach $^{16}$ (hereafter TD(A)-DHFT). This correction to excitation energy is scaled by the weight of the second order (MP2) correlation term in the ground state energy expression. Using this formalism several authors have applied DHs to compute vertical excitation energies of organic molecules up to medium size ${ }^{17-20}$. Recently, scaling techniques ${ }^{21,22}$ have been applied to both increase the TD(A)-DHFT accuracy and reduce associated computational cost (for instance, by neglecting spin opposite terms) ${ }^{18,23}$.

Since the results obtained are extremely encouraging in term of accuracy, in the present paper we present the implementation and benchmarking of TD(A)-DFT approaches focusing on the 
analysis of their performance for the description of through space charge-transfer (CT) excitations which are well known to be very problematic to be described using standard global hybrid approaches ${ }^{24-29}$.

Two different families of functionals will be compared: those derived imposing the fulfillment of some theoretical constraints, and those based on a more empirical fitting approach. For each of these families the performance of pure (GGA), hybrid (global and range separated hybrids) or double-hybrid functionals will be firstly assessed on standard benchmark and next analyzed using a set of charge transfer dimers, recently investigated by Baer and collaborators, as test case ${ }^{28}$.

The paper is structured as follows: after a brief description of the CIS(D) correction in the context of the Time Dependent implementation of DH functionals realized in the Gaussian code (section 2) and of the overall computational details (section 3), the implementation is validated is section 4 using two standard benchmark sets (hereafter named as Thiel and RLex80-EX7-0) before the results obtained on the charge transfer systems are discussed. In this case both results obtained in the gas phase and solution - here represented by a polarizable continuum model - will be discussed.

Overall the results we obtain show how DHs can be safely used to correctly describe CT excitations of medium sized molecules at still affordable costs both in the gas phase and in solution.

\section{Theory and implementation}

The description of electronic excited states can be approached in several different ways. Conceptually the easiest approach is based on the Configuration Interaction method truncated to include only singly excited determinants (CIS $)^{30}$. In this case the excited states are found by diagonalizing the Hamiltonian matrix that spans only the subspace generated by singly excited determinants. In its extreme simplicity CIS method lacks an explicit treatment of the electronic correlation of the ground (reference) determinant. This is often recovered, at a much greater computational cost, by including higher order excited determinants, such as in the case of the CISD method where doubly excited determinants are included. The CIS method can thus be considered the entry level approach for the treatment of excited states, just as the HF method is the basic model to study the ground state electronic structure. Following this line of development, the same considerations that lead to the use of second order MøllerPlesset perturbation theory (MP2), to include correlation effects at the HF ground state level, 
can be applied to the CIS method. Indeed, an MP2 correction of CIS states was firstly proposed by Foresman et al. ${ }^{31}$.

According to the resulting CIS-MP2 method, the excited state energies depend on two kinds of perturbations applied on the ground state: the static electron-electron correlation operators $T_{n}$ (where $n$ classifies the number of electrons considered in the excitation) and the time dependent ones $U_{n}$. Each one of the $U_{n}$-s should be considered as the first order developing term of the related static operator $T_{n}$. For example, at the second order in perturbation theory these operators assume the form:

$$
\begin{aligned}
& T_{2} \Phi_{0}=\frac{-1}{4} \sum_{i j a b} \quad a_{i j}^{a b} \Phi_{i j}^{a b}=\frac{-1}{4} \sum_{i j a b} \frac{\left\langle\Phi_{i j}^{a b}|V| \Phi_{0}\right\rangle}{\Delta_{i j}^{a b}} \Phi_{i j}^{a b}=\frac{-1}{4} \sum_{i j a b} \quad \frac{\langle i j \mid a b\rangle}{\Delta_{i j}^{a b}} \Phi_{i j}^{a b} \\
& U_{2} \Phi_{0}=\frac{-1}{4} \sum_{i j a b} b_{i j}^{a b} \Phi_{i j}^{a b}=\frac{-1}{4} \sum_{i j a b} \quad \frac{\left\langle\Phi_{i j}^{a b}|V| U_{1} \Phi_{0}\right\rangle}{\Delta_{i j}^{a b}-\omega} \Phi_{i j}^{a b}
\end{aligned}
$$

where $\mathrm{V}$ is the perturbation potential due to the electron correlation, $a$ and $b$ are the amplitudes relative to $T$ and $U$ operators respectively, $\Delta$ is an energy difference between $\mathrm{HF}$ orbital energies and, as usual, the $i, j, .$. indicate occupied orbitals whereas $a, b, .$. stand for virtual ones. In its first formulation the excited energy $E^{C I S}$ with the $M P 2$ correction is then written as

$E^{C I S-M P 2}=\left\langle\Phi_{C I S}|V| U_{2} \Phi_{0}\right\rangle+\left\langle\Phi_{C I S}|V| U_{3} \Phi_{0}\right\rangle=\frac{-1}{4} \sum_{i j a b} \quad \frac{\left(u_{i j}^{a b}\right)^{2}}{\Delta_{i j}^{a b}-\omega}-\frac{1}{36} \sum_{i j k a b c} \quad \frac{\left(u_{i j k}^{a b c}\right)^{2}}{\Delta_{i j k}^{a b c}-\omega}$

Where the $\Phi_{C I S}$ excited state mixes with states that came from the spaces of the double $\left(U_{2} \Phi_{0}\right)$ and triple $\left(U_{3} \Phi_{0}\right)$ excitations trough the perturbation to rise the direct and indirect terms, respectively. The indirect terms indeed describe triple excitation determinantal states that are created by the MP2 perturbation (a double excitation) of a single excited state. These are the terms responsible for the lack of self-consistency and the main computational burden, with an algorithmic complexity of $O\left(N^{6}\right)$.

An appealing approximation of the indirect terms in Eq.(3), based on the comparison of CISD and CCSD expansion, involves replacing the $U_{3}$ operator with the product $T_{2} U_{1}$, i.e. assuming that the MP2 amplitudes of pairs of electrons not involved in the excitations remain unchanged. The resulting CIS(D) method ${ }^{16}$ and has the following total energy expression

$$
E^{C I S(D)}=E^{M P 2}-\frac{1}{4} \frac{\sum_{i j a b}\left(u_{i j}^{a b}\right)^{2}}{\Delta_{i j}^{a b}-\omega}+\sum_{i a} b_{i}^{a} v_{i}^{a}
$$


then the (D)-corrected transition energy $\omega^{C I S(D)}$ has to be computed with respect to the MP2 energy of the ground state as $\omega^{C I S(D)}=E^{C I S(D)}-E^{M P 2}$. In Eq.(3) and Eq.(4) the quantities $u_{i j}^{a b}$ and $v_{i}^{a}$ are defined as

$$
\begin{gathered}
u_{i j}^{a b}=\sum_{c}\left(\langle a b \| c j\rangle b_{i}^{c}\right)-\left(\langle a b \| c i\rangle b_{j}^{c}\right)+\sum_{k}\left(\langle k a \| i j\rangle b_{k}^{b}\right)-\left(\langle k b \| i j\rangle b_{k}^{a}\right) \\
v_{i}^{a}=\frac{1}{2} \sum_{j k b c}\langle j k \| b c\rangle\left(b_{i}^{b} a_{j k}^{c a}+b_{j}^{a} a_{i k}^{c b}+2 b_{j}^{b} a_{i k}^{a c}\right)
\end{gathered}
$$

The computational complexity of the CIS(D) method is reduced to $O\left(N^{5}\right)$ with respect to CISMP2, but more importantly size-consistency is recovered.

During the twenty five years since the introduction of the CIS(D) method in 1994, timedependent Density Functional Theory (TD-DFT) has emerged as the method of choice for the study of excited states, by providing a convenient compromise between accuracy and computational cost. In its full form, TD-DFT is a method related to the random phase approximation (RPA), and as such it involves both single excitations and deexcitations. However, following the Tamm-Dancoff approximation, TD(A)-DFT is equivalent to perform a CIS calculation starting from the Kohn-Sham ground state determinant, involving only single excitations. On the other hand, while TD(A)-DFT was being recognized as the method of choice for the study of excited states, Grimme ${ }^{6}$ proposed an approach to apply the usual MP2 perturbative correction to a Kohn-Sham ground state determinant. This idea led to a new class of density functional approximations (DFA) termed double hybrids, which are characterized by the energy expression

$$
E_{\mathrm{xc}}^{\mathrm{DH}}(\rho)=a_{x} E_{x}^{\mathrm{HF}}+\left(1-a_{x}\right) E_{x}^{\mathrm{DFA}}(\rho)+\left(1-a_{c}\right) E_{c}^{\mathrm{DFA}}(\rho)+a_{c} E_{c}^{M P 2}
$$

where the $x$ and the $c$ subscripts indicate exchange and correlation contributions, respectively, while $E^{H F}$ is the usual Hartree-Fock exchange energy and $E_{c}{ }^{M P 2}$ is the correlation energy correction according to the MP2 approach. The addition of this last contribution to the DFT energy expression increases the computational cost to $O\left(N^{5}\right)$ but allows for an enhanced accuracy of the description of the electronic ground state, especially in those cases known to be problematic for standard DFAs ${ }^{13,14}$.

The combination of double hybrid DFA and a second order perturbative correction equivalent to CIS(D) can be readily achieved in the context of TD(A)-DFT, while for TD-DFT the most practical approach still involves just the use of the excitation single amplitudes in the (D) correction $^{23}$. This can be done essentially introducing the correction to the contribution stemming from the DFA part $\left(\Omega_{T D A-G H}\right.$ in Eq.(8)) for the same amount the MP2 is involved in the ground state energy of Eq.(7) end obtaining that:

$$
\Omega_{T D A-D H}=\Omega_{T D A-G H}+a_{c} \omega^{C I S(D)}
$$


Also in this context the computational scaling and the performance of double hybrids can be improved if a separate scaling for the same-spin (SS) and the opposite-spin (OS) contributions to the $E_{c}^{M P 2}$ corrections is applied ${ }^{23,32}$. Similarly, different scaling factors for the SS and SO terms has also been proposed and used to tune excited state calculations using the CIS(D) correction $^{32}$. Moreover, an additional parameter $\lambda$ can be introduced, which scales the TD(A)DFT transition energy in the denominator of the second term on the right-hand side of equation (4) ${ }^{23}$. In the present work any scaling of the $U_{1}$ and the $T_{2}$ terms or $\lambda$ values other than one, were employed only for purpose of validating our implementation against results published in the literature. To the best of the authors' understanding the (D) correction to TD(A)-DFT calculations using double-hybrid DFAs, has been until now implemented only in codes that make systematic use of the resolution-of-the-identity (RI) approximation ${ }^{33}$ such as $\mathrm{ORCA}^{34}$, and Q-Chem ${ }^{35}$. Despite the nonnegligible saving in computational cost, the use of the RI approximation relies on the assumption that the auxiliary expansion basis set being used is complete. However, "there is no guarantee that the approximation becomes uniformly better as the $[\ldots]$ completeness of the basis increases or that error in the approximation is known a priori" 33 .

The novelty of this work consists in our choice to evaluate the (D) correction within the code that implements the MP2 semi-direct algorithm, which is used in most practical cases by the Gaussian program ${ }^{36}$ to compute $E^{M P 2}$. Such algorithm, originally described in reference 37 , does not rely in the RI approximation. One important feature of our implementation is the ability to compute the (D) correction to a list of $N$ states at a cost of about $(N+1)$ MP2 calculation. All required quantities are assembled from AO two electron integrals which are generated on the-fly in a direct fashion. In the first pass, the ground state DH-DFA energy is computed and at the same time we can also evaluate the contribution to the last term on the right hand side of Eq.(4) arising from the whole $v_{i}^{a}$ terms that contribute to the (D) correction for all the $N$ states under consideration. This can be explained rewriting this last term inserting Eq.(6) in Eq.(4) and recognizing the similarity with Eq.(12), (13) and (14) in reference 38 in the following manner:

$$
\sum_{i a} b_{i}^{a} v_{i}^{a}=\frac{1}{2} \sum_{i a b} b_{i}^{a}(S 2)_{a b} b_{i}^{b}+\frac{1}{2} \sum_{i j a} b_{i}^{a}(S 1)_{i j} b_{j}^{a}+\frac{1}{2} \sum_{i k a c} b_{i}^{a} a_{i k}^{a c} F_{k c}
$$

where the quantities $(S 1)_{i j}$ and $(S 2)_{a b}$ are defined as

$$
(S 1)_{i j}=\sum_{k b c}\langle j k \| b c\rangle a_{i k}^{c b}(S 2)_{a b}=\sum_{j k c}\langle j k \| b c\rangle a_{j k}^{c b}
$$

and they are evaluated using the existing MP2 gradient $\operatorname{code}^{38}$. This allows to save a lot of computations because, for example we do not need to form the two-electron integrals with 
three virtual indexes during the evaluation of $(S 2)_{a b}$. To continue, the last term in Eq.(9) involves a Fock matrix including Coulomb and (full) exchange contributions only,

$$
F_{k c}=\sum_{j b}\langle j k \| b c\rangle b_{j}^{b}
$$

which is produced in a direct fashion from the single amplitudes transformed to the AO basis. This same term is evaluated for all the $N$ states as the ground state $E^{M P 2}$ is computed as usual by multiplying the $a_{i j}^{a b}$ double amplitudes with the fully transformed $\langle a b \| i j\rangle$ integrals in the MO basis.

Following this first pass, $N$ more MP2-like energy calculations are performed to compute instead the second term on the right hand side of Eq.(4) for each of the excited state under consideration. In fact, it can be shown that the required $u_{i j}^{a b}$ terms are double-like amplitudes and can be written as

$$
u_{i j}^{a b}=\langle a b \| \tilde{\imath} j\rangle+\langle a b \| i \tilde{\jmath}\rangle-\langle\tilde{a} b \| i j\rangle-\langle a \tilde{b} \| i j\rangle
$$

where the two-electron integrals in each term, are transformed on three indexes using the regular MO coefficients, while the remaining index, marked with the tilde, is transformed using a second set of MO coefficients pre-contracted with the single amplitudes,

$$
C_{\mu \tilde{\imath}}=\sum_{c} C_{\mu c} b_{i}^{c} C_{\mu \tilde{a}}=\sum_{j} C_{\mu j} b_{j}^{a} .
$$

This means that the difference between a regular two-electron integral and the tilded ones in Eq.(12) resides only in one of the four quarter transformation where, instead of transforming the AO to one of the MO, we are transforming to one of the excitation orbitals usually a combination of occupied and virtual MO. Bearing in mind that an excitation orbital belongs to the same space spanned by the regular MO it is straightforward to employ the same algorithm used to compute the $E^{M P 2}$ energy to evaluate the second term on the right hand side of Eq.(4). It is only necessary to recognize that we form the $u_{i j}^{a b}$ amplitudes instead of the usual $a_{i j}^{a b}$ double amplitudes, and that we have include the TD(A)-DFT transition energy $\Omega_{T D A-G H}$ of the excited state in the denominator. The resulting algorithm is then a generalization of the one initially proposed in reference 37. Explicitly, in the first quarter transformation a second kind of partially transformed integrals $\langle\tilde{\imath} v \| \lambda \sigma\rangle$ is produced in addition to the $\langle i v \| \lambda \sigma\rangle$, while at the end of the second quarter transformation three kinds of half-transformed integrals are made available, namely $\langle\tilde{\imath} v|| \lambda \sigma\rangle,\langle i \tilde{a}|| \lambda \sigma\rangle$ and $\langle i a|| \lambda \sigma\rangle$. The third and fourth quarter transformations is then run two times on $\langle i a|| \lambda \sigma\rangle$ to produce two sets of fully transformed integrals $\langle i a|| \tilde{j} b\rangle$ and $\langle i a|| \lambda \sigma\rangle$. On the other hand the usual second half transformations is applied to $\langle\tilde{\imath} a|| \lambda \sigma\rangle$ and $\langle i a|| \lambda \sigma\rangle$ to produce $\langle\tilde{\imath} a|| j b\rangle$ and $\langle i \tilde{a}|| j b\rangle$, respectively. At this point, all the basics tilded integrals are available and, as the regular MP2 energy code does, it is possible to build 
routinely their anti-symmetrized combinations appearing in Eq.(12), to calculate the $u_{i j}^{a b}$ amplitudes. These amplitudes are then added up with their relative weight depending on orbital energy difference to form the second term on the right hand side of Eq.(4).

The algorithm described above to calculate the (D) correction on one excited state is characterized by the same computational cost scaling as that of the ground state algorithm employed for MP2 and double hybrid DFAs. Then a linear increase in cost is observed as a function of the number of excited states which are considered to be corrected.

\section{Computational details}

All the calculations were performed with a modified version of the Gaussian development $\operatorname{code}^{36}$. Ground state structural optimization were performed at the MP2 $2^{39} / 6-31 G(d){ }^{40,41}$ level of theory for all molecules belonging to the Thiel ${ }^{42}$ and RLex80-EX7-0 ${ }^{20,43}$ subsets. Optimized structures for the Baer set (obtained at the B3LYP ${ }^{44}$ level with cc-pVDZ and augcc-pVDZ ${ }^{45}$ ) were taken from the reference 28 .

Vertical singlet excited states were computed at the TD-DFT level using different exchangecorrelation functionals namely: BLYP ${ }^{46,47}$, B3LYP ${ }^{44,48}, \mathrm{CAM}^{\mathrm{B} 3 L Y \mathrm{PP}^{49}}, \mathrm{PBE}^{50}, \mathrm{PBE}^{51,52}$, LC-PBE $^{53}$; the B2-PLYP ${ }^{6}$, PBE0-DH ${ }^{12}$, and PBE-QIDH ${ }^{11}$ in the case of the Thiel and RLex80-EX7-0 sets. The TZVP basis ${ }^{54}$ was used both for the Thiel and the RLex80-EX7-0 set. For selected molecules of this latter the def2-TZVP triple- $\zeta$ basis set ${ }^{55}$ used in the original publication of Bremond et al. ${ }^{20}$ was also tested and the corresponding results are reported in SI. In the case of the Baer Set, PBE, PBE0, LC-PBE, and PBE-QIDH functionals were considered using the cc-pVDZ and aug-cc-pVDZ basis sets. All calculations involving DH functionals were performed at the TDA level to allow comparison with previous work.

Solvent effects (methylene chloride) included using an implicit solvation model as implemented in the Gaussian code $\left(\mathrm{C}-\mathrm{PCM}^{56}\right)$.

\section{Results and discussion}

In order to validate our implementation, we firstly computed the excitation energies of the molecules belonging to the Thiel set for which DHs results can be found in literature ${ }^{17}$ and more recently ameliorated by the use of scaling techniques of spin-component and spinopposite (SCS/SOS) of electron-pair contributions to the nonlocal correlation components ${ }^{23}$. The set contains the 28 molecules reported in SI classed, following the original paper of Thiel, in 4 groups: 1) unsaturated hydrocarbons (7 molecules); 2) aromatic hydrocarbons and heterocycles (11 molecules); 3) aldehydes, ketones and amides (6 molecules), and 4) nucleobases (4 molecules). Detailed data (that are computed transition energies) for each 
group can be found in Supporting Information while the resulting Mean Absolute Deviations (MADs) are reported in Table 1.

As expected, the obtained results are completely consistent to the previously reported data ${ }^{17}$ for what concerns the B2-PLYP functional with a computed MAD of $0.24 \mathrm{eV}$ to be compared with the $0.22 \mathrm{eV}$ result reported in literature, thus validating our current implementation. Comparing the two families of functionals, that are those resting on the Becke exchange and LYP correlation with those mixing PBE exchange and correlation, the same general trend can be observed. Introducing exact exchange (B3LYP and PBE0) enhances the performances with respect to parent GGAs (BLYP and PBE) especially in the case of Group 3 and Group 4 molecules. This systematic improvement of performance is not observed when going from these global hybrids (B3LYP and PBE0) to the corresponding range separated hybrids (CAMB3LYP and LC-PBE) for which the agreement with the reference data is generally worse with few exceptions being represented by CAM-B3LYP performance for group 1 and group 3 molecules. Indeed, in the case of the PBE family the range separated LC-PBE shows a larger total MAD with respect to the corresponding global hybrid PBE0 $(0.63 \mathrm{eV}$ with respect to $0.30 \mathrm{eV})$.

On the other hand, all double hybrid functionals represent at best only a slight improvement with respect to global hybrid with a total MAD reducing from $0.35 \mathrm{eV}$ to $0.26 \mathrm{eV}$ (from B3LYP to B2-PLYP). Of note in the case of the PBE family both the PBE-QIDH and PBE0DH performance is slightly worse $(0.40 \mathrm{eV})$ than that of PBE0 $(0.30 \mathrm{eV})$ essentially due to the extremely good agreement with the reference data observed for the PBE0 functional for the molecules belonging to group 2 and group 4 with a MAD of $0.25 \mathrm{eV}$ and $0.18 \mathrm{eV}$, respectively.

All these general trends are in agreement with previously reported investigations ${ }^{17,20}$. Worth of discussion are indeed the results labelled as "PBE-QIH" in Table 1. These data correspond to the MADs associated to excitation energies computed at TD(A)-DFT level using the PBEQIDH functional but not including the doubles corrections. Comparison between the PBEQIH and PBE-QIDH MADs thus allow to evaluate directly the magnitude of these corrections. Clearly due to the large percentage of HF exchange, the PBE-QIH functional overestimates the transition energies providing very large MAD independently of the group of molecules considered with MADs ranging from $0.84 \mathrm{eV}$ for group 1 to $1.01 \mathrm{eV}$ for group 4 . Indeed, in this case the corrections $\left(a_{c} \omega^{C I S(D)}\right)$ are very large ranging from $0.15 \mathrm{eV}$ to roughly $0.70 \mathrm{eV}$, thus highlighting the importance of the correct treatment of perturbative contributions in DH functionals. 
To further test our implementation the seven molecules belonging to the RLex80-EX7-0 set were also considered. This benchmark set, depicted in Figure 1, was recently specifically developed to enable the fast evaluation of the performance of exchange correlation functionals for excited states. ${ }^{20}$ Data reported in Table 2 are obtained using the TZVP basis set for all functionals but the results and conclusions are comparable to those obtained using the $6-31+G(d)$ basis set for GGA and hybrid functionals and the def2-TZVP basis for DH functionals as suggested in the original paper, reported for selected systems in SI.

Compared to Thiel set, the RLex80-EX7-0 includes also transitions with a sizable intramolecular Charge Transfer character (CT) such as that of the molecule 74 (Figure 1), a push-pull chromophore, or of the molecule $\mathbf{7 5 .}$

Overall the same trends already observed for the Thiel set can be noted (Table 2). If global hybrids (B3LYP and PBE0) still represent the best cost to accuracy compromise, double hybrids, and particularly B2-PLYP, are extremely well performing with total MAD ranging from $0.11 \mathrm{eV}$ (B2-PLYP) to 0.19 and $0.21 \mathrm{eV}$ for PBE-QIDH and PBE0-DH, respectively. Of note all three DH functionals perform very well for molecules $\mathbf{7 4}$ and $\mathbf{7 5}$, that are those presenting transitions of partial CT character. For molecule $\mathbf{7 4}$ the effect of the inclusion of the perturbative correction is particularly large, as can be seen comparing the MAD computed for the PBE-QIH $(1.01 \mathrm{eV})$ and PBE-QIDH $(0.49 \mathrm{eV})$. Indeed, in such a case the inclusion of a larger percentage of HF exchange in the PBE-QIH it is not sufficient to recover the correct transition energy. Of note PBE0 is providing the smallest error and B3LYP is also showing an error of only $0.14 \mathrm{eV}$.

Nonetheless if both B3LYP and PBE0 provide very accurate intramolecular CT excitation energies in the case of molecule $\mathbf{7 4}$ and $\mathbf{7 5}$, it is well known that these global hybrid functionals fail to reproduce through space CT excitations.

To test the performance of DH functionals in the prediction of through-space CT excitations, the set of molecules recently proposed by Baer and collaborators ${ }^{28}$ was considered in the case of the PBE family of functionals (PBE, PBE0, LC-PBE and PBE-QIDH). This set contains a number of dimers composed by an aromatic molecule (benzene, toluene, o-xylene, naphthalene and substituted anthracene) acting as a donor, and tetracyanoethylene (TCNE) acting as an acceptor. For these molecules accurate experimental data both in the gas phase and/or in solution can be found in literature ${ }^{58,59}$. Of note we consider here only the effect of the functional on excitation energies, the geometry of each dimer being kept fixed at the B3LYP-optimized one as in the original paper of $\mathrm{Baer}^{28}$. 
First the results obtained for the Ar-TCNE dimers (Ar=benzene, toluene, o-xylene and napthatalene) will be considered since for these systems experimental data both in the gasphase and in methylene chloride solution are available ${ }^{58}$.

As reported in Table 3, for all dimers the first transition corresponds, as expected, to a HOMO-LUMO excitation, the HOMO being centered on the aryl donor and the LUMO on the TCNE acceptor. Analogously, for all systems also the second transition (of HOMO-1 to LUMO character) corresponds to an intermolecular $\pi_{\mathrm{Ar}}-\pi *_{\mathrm{TCNE}} \mathrm{CT}$ excitation. These two transitions are generally non-degenerate their difference in transition energies increasing with the size of the aromatic systems up to a value of $0.8 \mathrm{eV}$ in the case of the naphthalene. This trend is general roughly independently of the functional considered and of the presence or absence of the solvent. Starting from the third electronic transition, different functionals provide different ordering of the electronic transitions, pure functional predicting essentially a CT excitation of $n_{A r}-\pi *$ TCNE character while global, range-separated and double hybrids are predicting a local (and intense) $\pi_{\mathrm{TCNE}}-\pi^{*}{ }_{\mathrm{TCNE}}$ excitation. In the case of the naphthalene-TCNE system all functionals predict a third intermolecular CT excitation of $\pi_{\mathrm{Ar}}-\pi *_{\mathrm{TCNE}}$ character.

In order to evaluate the error in computed transition energies, since the reference data are experimental, two different MADs have been reported in Table 3. The first $\left(\mathrm{MAD}_{\mathrm{f}}\right)$ considering always the first CT excitation with the largest oscillator strength and the second one $\left(\mathrm{MAD}_{1 \mathrm{st}}\right)$ considering always the first computed transition independently of the associated intensity. The transition energies considered in the first case are reported in bold face in Table 3. Of note the computed oscillator strengths are, in agreement with the experimental data, relatively small and -using the basis set here considered, i.e. cc-pVDZ- not necessarily converged as already noted by Baer et al. ${ }^{28}$ Nonetheless the use of the larger aug-cc-pVDZ does not significantly change the general trend here reported (see SI for corresponding data). Independently of the criteria used to compute the MAD, both in the gas phase and in solution, the smallest errors are obtained using the PBE-QIDH functional while extremely large errors are computed at the GGA (PBE) or global hybrid (PBE0) level. DHs are better performing also with respect to range-separated hybrids (LC-PBE). Of note the agreement with the experimental data increases in going from the gas-phase to solution data. This is due essentially to error cancellation since transition energies are basically underestimated at all levels of theory as it is the effect of the solvent, which is experimentally also red-shifting the transition energies. As consequence the underestimation of solvent effect compensates the 
underestimation in computed transition energies allowing to afford extremely low values for the computed MAD in solution in the case of PBE-QIDH $(0.16-0.23 \mathrm{eV})$.

Overall from the analysis of our data we can conclude that DHs represent a good alternative to adjusted range separated hybrids for the treatment of intramolecular charge transfer excitations provided that the double correction is correctly included. Indeed excitation energies of $3.8 \mathrm{eV}, 3.4 \mathrm{eV}, 3.0 \mathrm{eV}$ and $2.7 \mathrm{eV}$ were reported for Aryl-TCNE (Aryl = benzene, toluene, o-xylene, naphthalene, respectively) by Baer and collaborators ${ }^{28}$ using the BNL functional and of $3.9 \mathrm{eV}$, by Truhlar and collaborators, using the revM11 functional in the case of the benzene-TCNE system. ${ }^{57}$

To further confirm these finding, the set of substituted anthracene-TCNE dimers (Table 4) by Baer was also considered. In this case experimental data are only available in solution ${ }^{59}$. Contrary to Baer previous work ${ }^{28}$ but analogously to what reported in Table 3 , transition energies were thus directly computed in solution using a polarizable continuum model. Of note BNL energies in solution taken from Baer work are instead estimated subtracting 0.32 $\mathrm{eV}$ from gas-phase computed data, the $0.32 \mathrm{eV}$ shift being estimated as an average experimental gas-phase to solvent shift ${ }^{59}$ on the basis also of a previous theoretical work ${ }^{60}$. The general trend observed are, as expected, in line with those of the previously discussed ArTCNE systems with a large/slight underestimation of transition energies provided by the GGA/global hybrid functional (PBE/PBE0) and an overestimation of transition energy provided by the range-separated LC-PBE. The results obtained with the PBE-QIDH functional are indeed in very good agreement with the experimental data in solution but generally all red-shifted with the prediction obtained at BNL level of theory in the gas-phase (see Table S5). These data are consistent with the previously pointed out error compensation between the underestimation of gas to solvent shift and transition energies.

Overall when considering the overall performance of PBE-QIDH, this functional not only shows a very small MAD with respect to solution data $(0.14 \mathrm{eV})$, comparable to that of the BNL functional $(0.09 \mathrm{eV})$, but, more relevantly is able to correctly recover the ordering of computed energies as a function of the substituent with the only exception of the inversion between the 9-methyl and 9, 10 dimethyl substituent. Furthermore it should be underlined that, contrary to adjusted range separated functionals, in double hybrids no parameter is tuned on a specific class of compounds to reproduce its excited state properties making them more appealing for a general use.

\section{Conclusions}


The results reported in this paper show how a Double Hybrids DFAs can be an effective tool to predict excited states energies of molecular compounds of relatively large size. Indeed, the algorithm described and implemented is characterized by the same computational cost scaling as that of the ground state algorithm employed for MP2 and double hybrid DFAs, although a linear increase in cost is observed as a function of the number of states considered. From the point of view of the performance we showed that the use of double hybrids allows for a better description of through space charge-transfer excitations which are well known to be very problematic to be described with commonly used functionals, such as global hybrids.

In particular, our results show, beside the robustness of the implementation, that double hybrids enable to obtain results comparable to that of adjusted range separated functionals, with the relevant difference that for DHs no parameter is tuned on a specific class of compounds to reproduce its excited state properties thus making them more appealing for a general use.

\section{Acknowledgments}

I.C and C.M acknowledge funding from the European Research Council (ERC) under the European Union's Horizon 2020 research and innovation programme (CoG STRIGES grant agreement No 648558). I.C. and C.A. acknowledge the French National Research Agency, ANR ("E-StorIc" project : ANR-14-CE05-0002). 


\section{References}

1) Mardirossian, N. and Head-Gordon M. Mol. Phys. 2017, 119, 2315-2372.

2) Cohen, A.J. Mori-Sánchez, P. and Yang; W. Chem. Rev. 2012, 112, 289-320

3) Zhao, Y. and Truhlar, D. G. Acc. Chem. Res. 2008, 41, 157-167.

4) Görling, A and Levy, M. Phys Rev B 1993, 47, 13105-13113.

5) Görling, A and Levy, M. Phys Rev A 1994, 50, 196-204.

6) Grimme, S. J. Chem. Phys. 2006, 124, 034108

7) Zhao, Y.; Lynch, B. J. and Truhlar, D. G. J. Phys. Chem. A 2004, 108, 4786-4791.

8) Goerigk, L.; Grimme, S WIREs: Comput. Mol. Sci. 2014, 4, 576-600.

9) Sancho-García, J. C. and Adamo, C. Phys. Chem. Chem. Phys. 2013, 15, 14581-14594.

10) Toulouse, J.; Sharkas, K.; Brémond, E. and Adamo, C. J. Chem. Phys. 2011, 135, 101102.

11) Brémond, É.; Sancho-García, J. C.; Pérez-Jiménez, Á. J. and Adamo, C. J. Chem. Phys. 2014, 141, 031101-031104

12) Brémond, É. and Adamo, C. J. Chem. Phys. 2011, 135, 024106.

13) Goerigk, L. and Grimme, S. Phys. Chem. Chem. Phys., 2011,13, 6670-6688

14) Schwabe, T. and Grimme, S. Phys. Chem. Chem. Phys., 2006, 8, 4398-4401

15) Grimme, S. and Neese, F. J. Chem. Phys. 2007, 127, 154116.

16) Head-Gordon, M.; Rico, R.J.; Oumi, M. and Lee, T.J. Chem. Phys. Lett. 1994, 219, 21-29

17) Goerigk, L.; Moellmann, J. and Grimme, S. Phys. Chem. Chem. Phys., 2009, 11, 46114620

18) Goerigk, L. and Grimme, S. J. Chem. Phys. 2010, 132, 184103

19) Di Meo, F.; Trouillas, P.; Adamo, C. and Sancho-García, J.C. J. Chem. Phys. 2013, 139, 164104.

20) Brémond, E.; Savarese, M.; Pérez-Jiménez, A.J.; Sancho-García, J.C. and Adamo, C. J. Chem. Theory Comput., 2017, 13, 5539-5551.

21) Grimme, S. J. Chem. Phys. 2003, 118, 9095-9102.

22) Kozuch, S. and Martin, J.M.L. Phys. Chem. Chem. Phys., 2011, 13, 20104-20107,

23) Schwabe, T. and Goerigk, L. J. Chem. Theory Comput. 2017, 13, 4307-4323

24) Dreuw, A. and Head-Gordon, M. J. Am. Chem. Soc. 2004, 126, 4007-4016.

25) Magyar, R.J. and Tretiak, S. J. Chem. Theory Comput. 2007, 3, 976-987

26) Autschbach, J. ChemPhysChem 2009, 10, 1757-1760

27) Maitra, N.T. J. Phys. : Condens. Matter 2017, 29, 423001

28) Stein, T.; Kronik, L. and Baer, R. J. Am. Chem. Soc. 2009, 131, 2818-2820 
29) Jacquemin, D.; Mennucci, B. and Adamo, C. Phys. Chem. Chem. Phys. 2011, 13, 16987-16998.

30) Szabo, A.; Ostlund, N.S. Modern Quantum Chemistry: Introduction to Advanced Electronic Structure Theory, Dover Publications, New York, 1996.

31) Foresman, J.B.; Head-Gordon, M.; Pople, J.A. and Frisch, M.J. J. Phys. Chem 1992, 96, 135-149

32) Rhee, Y. M. and Head-Gordon, M., J. Phys. Chem.A 2007, 111, 5314-5326.

33) Feyereisen, M.; Fitzgerald, G. and Komornicki, A. Chem Phys Lett 1993, 208, 359-363.

34) Neese, F. WIREs Comput Mol Sci 2018, 8, e1327.

35) Shao, Y.; Gan, Z.; Epifanovsky, E.; Gilbert, A. T. B.; Wormit, M.; Kussmann, J.; Lange, A. W.; Behn, A.; Deng, J.; Feng, X.; Ghosh, D.; Goldey, M.; Horn, P. R.; Jacobson, L. D.; Kaliman, I.; Khaliullin, R. Z.; Kus, T.; Landau, A.; Liu, J.; Proynov, E. I.; Rhee, Y. M.; Richard, R. M.; Rohrdanz, M. A.; Steele, R. P.; Sundstrom, E. J.; Woodcock, H. L., III; Zimmerman, P. M.; Zuev, D.; Albrecht, B.; Alguires, E.; Austin, B.; Beran, G. J. O.; Bernard, Y. A.; Berquist, E.; Brandhorst, K.; Bravaya, K. B.; Brown, S. T.; Casanova, D.; Chang, C.M.; Chen, Y.; Chien, S. H.; Closser, K. D.; Crittenden, D. L.; Diedenhofen, M.; DiStasio, R. A., Jr.; Do, H.; Dutoi, A. D.; Edgar, R. G.; Fatehi, S.; Fusti-Molnar, L.; Ghysels, A.; Golubeva-Zadorozhnaya, A.; Gomes, J.; Hanson-Heine, M. W. D.; Harbach, P. H. P.; Hauser, A. W.; Hohenstein, E. G.; Holden, Z. C.; Jagau, T.-C.; Ji, H.; Kaduk, B.; Khistyaev, K.; Kim, J.; Kim, J.; King, R. A.; Klunzinger, P.; Kosenkov, D.; Kowalczyk, T.; Krauter, C. M.; Laog, K. U.; Laurent, A.; Lawler, K. V.; Levchenko, S. V.; Lin, C. Y.; Liu, F.; Livshits, E.; Lochan, R. C.; Luenser, A.; Manohar, P.; Manzer, S. F.; Mao, S.-P.; Mardirossian, N.; Marenich, A. V.; Maurer, S. A.; Mayhall, N. J.; Oana, C. M.; Olivares-Amaya, R.; O’Neill, D. P.; Parkhill, J. A.; Perrine, T. M.; Peverati, R.; Pieniazek, P. A.; Prociuk, A.; Rehn, D. R.; Rosta, E.; Russ, N. J.; Sergueev, N.; Sharada, S. M.; Sharmaa, S.; Small, D. W.; Sodt, A.; Stein, T.; Stuck, D.; Su, Y.-C.; Thom, A. J. W.; Tsuchimochi, T.; Vogt, L.; Vydrov, O.; Wang, T.; Watson, M. A.; Wenzel, J.; White, A.; Williams, C. F.; Vanovschi, V.; Yeganeh, S.; Yost, S. R.; You, Z.-Q.; Zhang, I. Y.; Zhang, X.; Zhou, Y.; Brooks, B. R.; Chan, G. K. L.; Chipman, D. M.; Cramer, C. J.; Goddard, W. A., III; Gordon, M. S.; Hehre, W. J.; Klamt, A.; Schaefer, H. F., III; Schmidt, M. W.; Sherrill, C. D.; Truhlar, D. G.; Warshel, A.; Xu, X.; Aspuru-Guzik, A.; Baer, R.; Bell, A. T.; Besley, N. A.; Chai, J.-D.; Dreuw, A.; Dunietz, B. D.; Furlani, T. R.; Gwaltney, S. R.; Hsu, C.-P.; Jung, Y.; Kong, J.; Lambrecht, D. S.; Liang, W. Z.; Ochsenfeld, C.; Rassolov, V. A.; Slipchenko, L. V.; Subotnik, J. E.; van Voorhis, T.; Herbert, J. M.; 
Krylov, A. I.; Gill, P. M. W.; Head-Gordon, M. Advances in molecular quantum chemistry contained in the Q-Chem 4 program package. Mol. Phys. 2015, 113, 184-215.

36) Frisch, M. J.; Trucks, G. W.; Schlegel, H. B.; Scuseria, G. E.; Robb, M. A.; Cheeseman, J. R.; Scalmani, G.; Barone, V.; Petersson, G. A.; Nakatsuji, H.; Li, X.; Caricato, M.; Marenich, A. V.; Bloino, J.; Janesko, B. G.; Gomperts, R.; Mennucci, B.; Hratchian, H. P.; Ortiz, J. V.; Izmaylov, A. F.; Sonnenberg, J. L.; Williams-Young, D.; Ding, F.; Lipparini, F.; Egidi, F.; Goings, J.; Peng, B.; Petrone, A.; Henderson, T.; Ranasinghe, D.; Zakrzewski, V. G.; Gao, J.; Rega, N.; Zheng, G.; Liang, W.; Hada, M.; Ehara, M.; Toyota, K.; Fukuda, R.; Hasegawa, J.; Ishida, M.; Nakajima, T.; Honda, Y.; Kitao, O.; Nakai, H.; Vreven, T.; Throssell, K.; Montgomery, J. A., Jr.; Peralta, J. E.; Ogliaro, F.; Bearpark, M. J.; Heyd, J. J.; Brothers, E. N.; Kudin, K. N.; Staroverov, V. N.; Keith, T. A.; Kobayashi, R.; Normand, J.; Raghavachari, K.; Rendell, A. P.; Burant, J. C.; Iyengar, S. S.; Tomasi, J.; Cossi, M.; Millam, J. M.; Klene, M.; Adamo, C.; Cammi, R.; Ochterski, J. W.; Martin, R. L.; Morokuma, K.; Farkas, O.; Foresman, J. B.; Fox, D. J. Gaussian Development Version, Revision J.1, Gaussian, Inc., Wallingford CT, 2018.

37) Frisch, M.J.; Head-Gordon, M. and Pople, J.A. Chem. Phys. Lett. 1990, 166, 281-289.

38) Frisch, M.J.; Head-Gordon, M. and Pople, J.A. Chem. Phys. Lett. 1990, 166, 275-280.

39) Møller, C. and Plesset, M.S. Phys. Rev., 1934, 46, 618-622

40) Ditchfield, R.; Hehre, W.J. and Pople, J.A. J. Chem. Phys., 1971, 54, 724-728.

41) Hehre, W.J.; Ditchfield, R. and Pople, J.A. J. Chem. Phys., 1972, 56, 2257-2261.

42) Schreiber, M.; Silva-Junior, M.R.; Sauer, S.P.A. and Thiel, W. J. Chem. Phys. 2008, 128, 134110.

43) Jacquemin, D.; Duchemin, I. and Blase, X. J. Chem. Theory Comput. 2015, 11, 53405359.

44) Becke, A. D. J. Chem. Phys. 1993, 98, 5648-5652

45) Dunning Jr., T.H. J. Chem. Phys. 1989; 90, 1007-1023

46) Becke, A. D. Phys. Rev. A 1998, 38, 3098-3100

47) Lee, C. T. ; Yang, W. T. and Parr, R. G. Phys. Rev. B 1988, 37, 785-789

48) Stephens, P. J.; Devlin, F. J.; Chabalowski, C. F. N. and Frisch, M. J. J. Phys. Chem. 1994, 98, 11623-11627.

49) Yanai, T.; Tew, D. P. and Handy, N. C. Chem. Phys. Lett. 2004, 393, $51-57$.

50) Perdew, J. P.; Burke, K. and Ernzerhof, M. Phys. Rev. Lett. 1996, 77, 3865-3868

51) Adamo, C. and Barone, V. J. Chem. Phys. 1999, 110, 6158-6170. 
52) Ernzerhof, M. and Scuseria, G. E. J. Chem. Phys. 1999, 110, 5029-5036.

53) Iikura, H.; Tsuneda, T.; Yanai, T. and Hirao, K. A J. Chem. Phys. 2001, 115, 3540-3544.

54) Schäfer, A.; Huber, C. and Ahlrichs, R. J. Chem. Phys. 1994, 100, 5829-5835.

55) Weigend, F. and Ahlrichs, R. Phys. Chem. Chem. Phys. 2005, 7, 3514-3305

56) Barone, V. and Cossi, M. J. Phys. Chem. A, 1998, 102, 1995-2001.

57) Verma, P.; Wang, Y.; Ghosh, S.; He, X. and Truhlar, D.G. J. Phys. Chem. A 2019, 123, 2966-2990

58) Hanazaki, I. J. Phys. Chem. 1972, 76, 1982-1989

59) Masnovi, J.M.; Seddon, E.A. and Kochi, J.K. Can. J. Chem. 1984, 62, 2552-2559.

60) Liao, M.-S.; Lu, Y.; Parker, V.D. and Scheiner, S. J. Phys. Chem. A 2003, 107, 89398948. 
Table 1. Computed Mean Absolute Deviation (MAD, in eV) for all functionals for the Thiel benchmark set. For groups definition refer to Figure 1. PBE-QIH results are obtained from PBE-QIDH TD(A)-DFT calculations not including the doubles correction (that is the $a_{c} \omega^{C I S(D)}$ term in eq. 8).

\begin{tabular}{cccccc}
\hline Functional & \multicolumn{5}{c}{ MAD $(\mathbf{e V})$} \\
\hline BLYP & Group 1 & Group 2 & Group 3 & Group 4 & Total \\
\cline { 2 - 5 } B3LYP & 0.72 & 0.39 & 0.70 & 0.86 & 0.61 \\
CAM-B3LYP & 0.60 & 0.24 & 0.36 & 0.29 & 0.31 \\
B2-PLYP & 0.54 & 0.29 & 0.44 & 0.26 & 0.35 \\
PBE & 0.31 & 0.29 & 0.21 & 0.16 & 0.26 \\
PBE0 & 0.78 & 0.38 & 0.68 & 0.86 & 0.62 \\
LC-PBE & 0.60 & 0.25 & 0.38 & 0.18 & 0.30 \\
PBE-QIH & 0.63 & 0.52 & 0.62 & 0.57 & 0.63 \\
PBE-QIDH $^{\mathrm{a}}$ & 0.54 & 0.84 & 0.77 & 1.01 & 0.88 \\
PBE0-DH $^{\mathrm{a}}$ & 0.39 & 0.46 & 0.29 & 0.34 & 0.40 \\
\hline
\end{tabular}

${ }^{\mathrm{a}}$ Within the Tamm-Dancoff approximation. 
Table 2. Relative and Mean Absolute Deviations (MAD, in eV) computed using the TZVP basis set for the molecules belonging to the RLex80-EX7-0 data set (figure 1). The reference CC2 values, taken from reference 43 , are also reported.

\begin{tabular}{|c|c|c|c|c|c|c|c|c|}
\hline & $01 \mathrm{Mol}$ & $08 \mathrm{Mol}$ & $33 \mathrm{Mol}$ & $41 \mathrm{Mol}$ & $52 \mathrm{Mol}$ & $74 \mathrm{Mol}$ & $75 \mathrm{Mol}$ & MAD \\
\hline BLYP & 0.57 & 0.3 & 1.06 & 0.98 & 0.34 & 0.72 & 1.16 & 0.73 \\
\hline B3LYP & 0.15 & 0.06 & 0.27 & 0.71 & 0.08 & 0.14 & 0.52 & 0.28 \\
\hline CAM-B3LYP & 0.00 & 0.19 & 0.15 & 0.42 & 0.2 & 0.41 & 0.2 & 0.22 \\
\hline B2-PLYPa & 0.09 & 0.04 & 0.02 & 0.27 & 0.01 & 0.08 & 0.23 & 0.11 \\
\hline PBE & 0.53 & 0.3 & 1.04 & 0.94 & 0.33 & 0.71 & 1.15 & 0.71 \\
\hline PBEO & 0.05 & 0.01 & 0.1 & 0.62 & 0.01 & 0 & 0.37 & 0.17 \\
\hline LC-PBE & -0.17 & 0.43 & 0.44 & 0.11 & 0.5 & 0.88 & 0.65 & 0.45 \\
\hline PBE-QIH $^{\mathrm{a}}$ & -0.32 & 0.74 & 0.72 & 0.1 & 0.64 & 1.01 & 0.49 & 0.57 \\
\hline PBE-QIDH $^{\mathrm{a}}$ & 0.02 & 0.21 & 0.29 & 0.07 & 0.19 & 0.45 & 0.12 & 0.19 \\
\hline PBE0-DH ${ }^{\mathrm{a}}$ & 0.06 & 0.28 & 0.25 & 0.24 & 0.22 & 0.35 & 0.08 & 0.21 \\
\hline $\mathrm{CC2}$ & 5.41 & 2.24 & 4.06 & 4.77 & 2.65 & 3.71 & 3.37 & \\
\hline
\end{tabular}

${ }^{\mathrm{a}}$ Within the Tamm-Dancoff approximation 
Table 3. Computed and experimental excitation energies $(\mathrm{eV})$ for Ar-TCNE systems. For the sake of clarity, only the three lowest electronic transitions are reported. In bold transitions that have been considered to compute the $\mathrm{MAD}_{\mathrm{f}}$ (see text for definition). Highlighted in yellow intermolecular $\pi_{\mathrm{Ar}}-\pi *_{\mathrm{TCNE}} \mathrm{CT}$ transitions; in green intermolecular $\mathrm{n}_{\mathrm{Ar}}-\pi *_{\mathrm{TCNE}} \mathrm{CT}$ transitions; in pink local $\pi_{\mathrm{TCNE}}-\pi *_{\mathrm{TCNE}}$ excitations and in grey local $\pi_{\mathrm{Ar}}-\pi * \mathrm{Ar}$ excitations.

\begin{tabular}{|c|c|c|c|c|c|c|c|c|c|c|}
\hline TCNE- & $E$ & fosc & $\mathrm{E}$ & f osc & $\mathrm{E}$ & f osc & $\mathrm{E}$ & fosc & $\frac{\text { PBE-QIDH }^{\mathrm{a}}}{\mathrm{E}}$ & $\frac{\exp }{E}$ \\
\hline $\mathrm{S} 0 \rightarrow \mathrm{S} 1$ & 1.42 & 0.00 & 2.13 & 0.00 & 4.16 & 0.00 & 3.55 & 0.00 & 3.09 & \multirow[b]{2}{*}{3.59} \\
\hline $\mathrm{S} 0 \rightarrow \mathrm{S} 2$ & 1.55 & 0.03 & 2.21 & 0.03 & 4.17 & 0.03 & 3.59 & 0.03 & 3.12 & \\
\hline $\mathrm{S} 0 \rightarrow \mathrm{S} 1$ & 1.37 & 0.04 & 1.94 & 0.03 & 3.81 & 0.03 & 3.28 & 0.04 & 2.78 & \multirow{3}{*}{3.36} \\
\hline $\mathrm{S} 0 \rightarrow \mathrm{S} 2$ & 1.43 & 0.00 & 2.10 & 0.00 & 4.07 & 0.00 & 3.49 & 0.00 & 3.00 & \\
\hline $\mathrm{S} 0 \rightarrow \mathrm{S} 3$ & 3.17 & 0.00 & 4.37 & 0.00 & 4.85 & 0.32 & 5.04 & 0.44 & 5.08 & \\
\hline \multicolumn{11}{|l|}{ Xylene } \\
\hline $\mathrm{S} 0 \rightarrow \mathrm{S} 1$ & 1.06 & 0.00 & 1.68 & 0.01 & 3.55 & 0.01 & 3.02 & 0.01 & 2.51 & 3.15 \\
\hline $\mathrm{S} 0 \rightarrow \mathrm{S} 1$ & 0.36 & 0.0004 & 1.07 & 0.0002 & 3.17 & 0.0003 & 2.48 & 0.0003 & 2.02 & \multirow{3}{*}{2.60} \\
\hline $\mathrm{S} 0 \rightarrow \mathrm{S} 2$ & 1.07 & 0.0000 & 1.83 & 0.0001 & 3.93 & 0.0033 & 3.27 & 0.0007 & 2.78 & \\
\hline $\mathrm{S} 0 \rightarrow \mathrm{S} 3$ & 2.03 & 0.0050 & 2.99 & 0.0047 & 4.78 & 0.0001 & 4.79 & 0.0066 & 4.02 & \\
\hline MAD $_{\mathrm{f}}$ & \multicolumn{2}{|c|}{1.99} & \multicolumn{2}{|c|}{1.37} & \multicolumn{2}{|c|}{$0.76_{5}$} & \multicolumn{2}{|c|}{0.23} & $0.39_{5}$ & \\
\hline MAD $_{1 \text { st }}$ & \multicolumn{2}{|c|}{2.12} & \multicolumn{2}{|c|}{3.16} & \multicolumn{2}{|c|}{0.50} & \multicolumn{2}{|c|}{0.09} & $0.57_{5}$ & \\
\hline
\end{tabular}




\begin{tabular}{|c|c|c|c|c|c|c|c|c|c|c|}
\hline \multicolumn{11}{|c|}{ solvent } \\
\hline & \multicolumn{2}{|c|}{ PBE } & \multicolumn{2}{|c|}{ PBE0 } & \multicolumn{2}{|c|}{ LC-PBE } & \multicolumn{2}{|c|}{ PBE-QIH $^{\mathrm{a}}$} & \multirow{2}{*}{$\begin{array}{l}\text { PBE-QIDH }^{\mathrm{a}} \\
\mathrm{E}\end{array}$} & \multirow{2}{*}{$\begin{array}{c}\exp ^{b} \\
E\end{array}$} \\
\hline TCNE- & $\mathrm{E}$ & fosc & $\mathrm{E}$ & fosc & $\mathrm{E}$ & f osc & $\mathrm{E}$ & fosc & & \\
\hline \multicolumn{10}{|l|}{ Benzene } & \multirow{4}{*}{3.22} \\
\hline $\mathrm{S} 0 \rightarrow \mathrm{S} 1$ & 1.42 & 0.0000 & 2.13 & 0.0000 & 4.11 & 0.0000 & 3.53 & 0.0000 & 3.07 & \\
\hline $\mathrm{S} 0 \rightarrow \mathrm{S} 2$ & 1.56 & 0.0433 & 2.20 & 0.0403 & 4.12 & 0.0388 & 3.55 & 0.0465 & 3.09 & \\
\hline $\mathrm{S} 0 \rightarrow \mathrm{S} 3$ & 3.22 & 0.0000 & 4.27 & 0.3716 & 4.75 & 0.4343 & 4.90 & 0.5629 & 4.93 & \\
\hline \multicolumn{10}{|l|}{ Toluene } & \multirow{4}{*}{3.05} \\
\hline $\mathrm{S} 0 \rightarrow \mathrm{S} 1$ & 1.40 & 0.0582 & 1.95 & 0.0532 & 3.76 & 0.0474 & 3.25 & 0.0585 & 2.75 & \\
\hline $\mathrm{S} 0 \rightarrow \mathrm{S} 2$ & 1.45 & 0.0001 & 2.11 & 0.0000 & 4.04 & 0.0000 & 3.48 & 0.0000 & 3.00 & \\
\hline $\mathrm{S} 0 \rightarrow \mathrm{S} 3$ & 3.14 & 0.0000 & 4.26 & 0.3518 & 4.74 & 0.4211 & 4.90 & 0.5465 & 4.93 & \\
\hline \multicolumn{10}{|l|}{ Xylene } & \multirow{4}{*}{2.89} \\
\hline $\mathrm{S} 0 \rightarrow \mathrm{S} 1$ & 1.19 & 0.0116 & 1.77 & 0.0215 & 3.56 & 0.0309 & 3.07 & 0.0335 & 2.55 & \\
\hline $\mathrm{S} 0 \rightarrow \mathrm{S} 2$ & 1.50 & 0.0643 & 2.03 & 0.0456 & 3.84 & 0.0274 & 3.33 & 0.0381 & 2.81 & \\
\hline $\mathrm{S} 0 \rightarrow \mathrm{S} 3$ & 3.11 & 0.0002 & 4.22 & 0.2216 & 4.73 & 0.4084 & 4.89 & 0.5313 & 4.91 & \\
\hline \multicolumn{10}{|c|}{ Naphtalene } & \multirow{4}{*}{2.26} \\
\hline $\mathrm{S} 0 \rightarrow \mathrm{S} 1$ & 1.22 & 0.1011 & 1.54 & 0.0882 & 3.18 & 0.0675 & 2.63 & 0.0875 & 2.12 & \\
\hline $\mathrm{S} 0 \rightarrow \mathrm{S} 2$ & 1.60 & 0.0000 & 2.13 & 0.0000 & 3.95 & 0.0011 & 3.38 & 0.0003 & 2.86 & \\
\hline $\mathrm{S} 0 \rightarrow \mathrm{S} 3$ & 2.56 & 0.0132 & 3.25 & 0.0115 & 4.71 & 0.2609 & 4.84 & 0.1554 & 4.59 & \\
\hline $\mathbf{M A D}_{\mathbf{f}}$ & \multicolumn{2}{|c|}{1.43} & \multicolumn{2}{|c|}{0.92} & \multicolumn{2}{|c|}{0.80} & \multicolumn{2}{|c|}{0.335} & 0.16 & \\
\hline $\mathbf{M A D}_{1 \text { st }}$ & \multicolumn{2}{|c|}{1.55} & \multicolumn{2}{|c|}{1.01} & \multicolumn{2}{|c|}{0.80} & \multicolumn{2}{|c|}{0.265} & 0.23 & \\
\hline
\end{tabular}

a) Within the Tamm-Dancoff approximation. b) from reference 58. 
Table 4. Computed and experimental excitation energies (in eV) for a series of substituted anthracene-TCNE systems.

\begin{tabular}{cccccccc}
\hline \multirow{2}{*}{ Substituent } & PBE & PBE0 & LC-PBE & PBE-QIH & PBE-QIDH $^{\mathrm{a}}$ & BNL $^{\mathrm{b}}$ & exp. $^{\mathrm{c}}$ \\
\cline { 2 - 8 } none & 1.38 & 1.47 & 2.47 & 2.09 & 1.58 & 1.82 & 1.73 \\
9-cyano & 0.65 & 1.02 & 3.12 & 2.42 & 1.90 & 2.03 & 2.01 \\
9-chloro & 1.16 & 1.27 & 2.56 & 2.08 & 1.58 & 1.82 & 1.74 \\
9-carbomethoxy & 1.06 & 1.22 & 2.66 & 2.15 & 1.64 & 1.84 & 1.84 \\
9-methyl & 1.26 & 1.34 & 2.31 & 1.93 & 1.42 & 1.71 & 1.55 \\
9-nitro & 0.92 & 1.43 & 2.68 & 2.50 & 1.98 & 2.12 & 2.03 \\
9,10-dimethyl & 1.52 & 1.59 & 2.24 & 2.03 & 1.53 & 1.77 & 1.44 \\
9-formyl & 0.85 & 1.26 & 2.72 & 2.20 & 1.7 & 1.95 & 1.9 \\
9-formyl 10-chloro & 0.83 & 1.25 & 2.83 & 2.30 & 1.79 & 1.96 & 1.96 \\
\hline MAD & $\mathbf{0 . 6 3}$ & $\mathbf{0 . 4 9}$ & $\mathbf{0 . 8 1}$ & $\mathbf{0 . 3 9}$ & $\mathbf{0 . 1 4}$ & $\mathbf{0 . 0 9}$ & \\
\hline
\end{tabular}

a) Within the Tamm-Dancoff approximation; b) From reference 59. c) From reference 28. 


\section{Figure captions}

Figure 1 Molecules of the RLex80-EX7-0 set ${ }^{20}$ considered in this work 

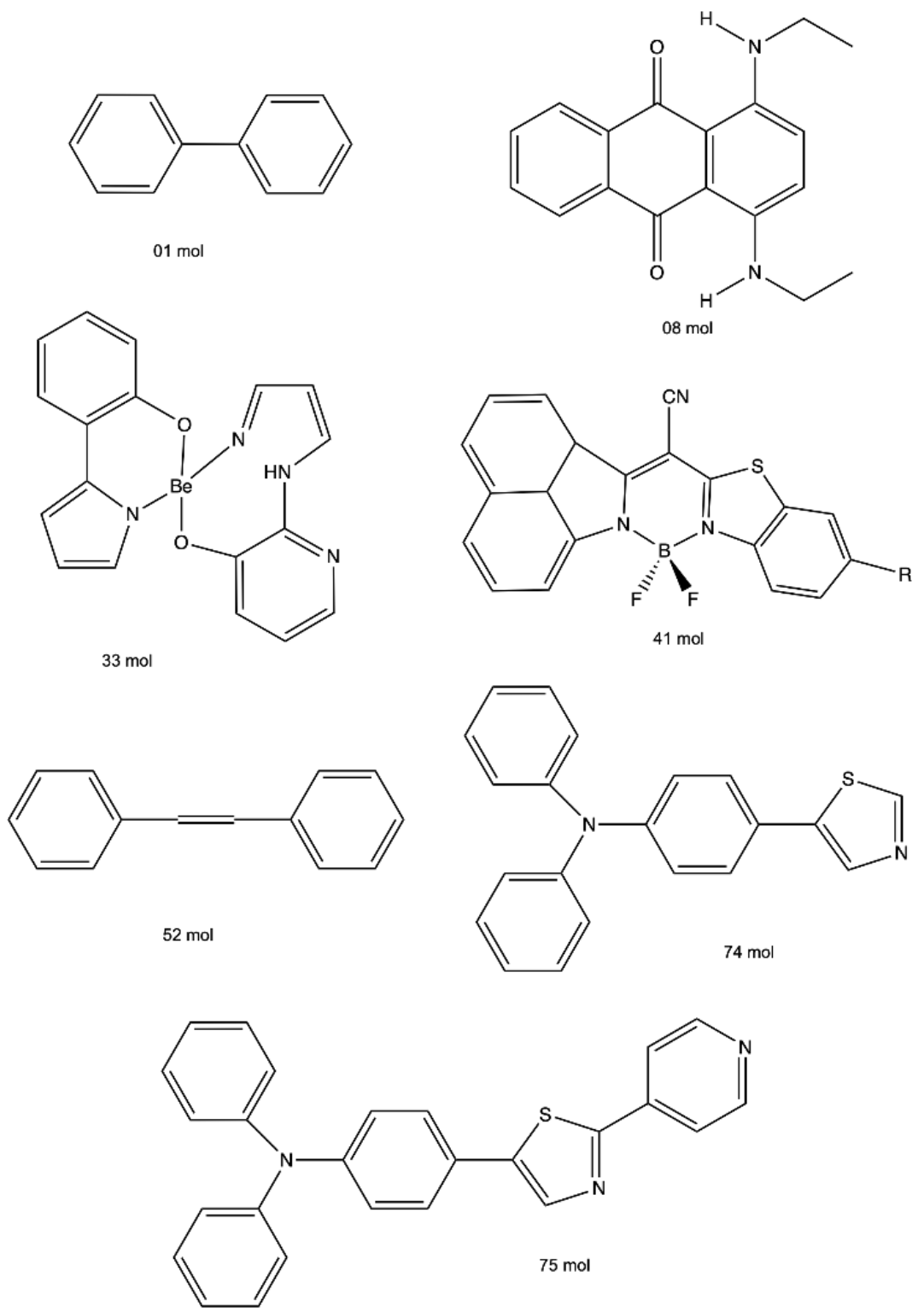

Figure 1 